\title{
Entwicklung eines Konzepts zum Lagermanagement mit Optimierungsansätzen auf Ebene eines neuen Regalbediengerätes
}

\author{
Development of a new Warehouse Management Concept with Optimization \\ Techniques concerning an Innovative Storage and Retrieval Machine
}

\author{
Bashir Salah \\ Marc Strehle \\ Bernd Noche \\ Lehrstuhl für Transportsysteme und Logistik \\ Fakultät für Ingenieurswissenschaften \\ Universität Duisburg-Essen
}

D ie Grundidee hinter dem neuen Regalbediengerät ist die Minimierung der zu bewegenden Last durch die Verwendung einer seilbasierten Stewart-GoughPlattform (SGP). Das klassische Regalbediengerät (RBG) wird dabei durch eine Plattform ersetzt, die an bis zu acht vorgespannten Seilen befestigt ist. Unterstützt wird diese Bauform durch eine abgestimmte Steuerungssoftware, die es ermöglicht die Vorteile des RBG optimal zu nutzen. Das hier mit Software vorgestellte Konzept regelt die Vorgehensweise zur Optimierung von Ein- und Auslagerungsaufträgen in einem Lagerregal, sowie die Optimierung der Fachbelegung in Zeiten ohne direkten Auftrag. Es beinhaltet dabei Mechanismen, die bestimmen wann Aufträge abgearbeitet werden, in welcher Reihenfolge und in welchen Kombinationen. Ziel ist es Ein- und Auslagerungsaufträge so zu kombinieren, dass möglichst effizient und schnell gearbeitet wird.

[Schlüsselwörter: TSP-Modul, Stewart-Gough Plattform(SGP), RBG-Modul, Algorithmus, Einzelspiel]

$\mathbf{T}$ he basic idea behind the proposed storage and retrieval machine is to minimize the mass to be moved by using a cable-based on Stewart-Gough Platform (SGP). Conventional storage and retrieval machines (SRMs) are replaced with a platform which is attached to up to eight pre-tensioned cables. The software is used to manage the transactions for the optimization of storage and retrieval orders in warehouse shelves, as well as the optimization of the inventory at any times without any direct orders. It includes mechanisms which determine when orders are processed, in which order, and in which combinations. The main goal is to combine storage and retrieval orders so it is worked as fast and efficient as possible.

[Keywords: TSP module, Stewart-Gough platform (SGP), SRM module, algorithm, single operation cycle]

\section{EINLEITUNG}

Die Grundidee hinter dem neuen Regalbediengerät ist die Minimierung der zu bewegenden Last durch die Verwendung einer seilbasierten SGP [Sal11]. Unter Einsatz mechatronischer Entwicklungsmethoden wird im Rahmen dieses Projektes ein innovatives System realisiert, das mittels computergesteuerten Seilwinden unter Einsatz modernster Regelungsverfahren Lasten schnell und energieeffizient befördert. Das klassische Regalbediengerät wird dabei durch eine Plattform ersetzt, die an acht vorgespannten Seilen befestigt ist. Der einfache mechanische Aufbau führt dazu, dass besonders wenige Massengewichte bewegt werden müssen. In Verbindung mit einer leistungsfähigen Echtzeitregelung und einem Algorithmus zur Optimierung von Ein- und Auslagerungsaufträgen in einem Lagerregal, sowie die Optimierung der Fachbelegung in Zeiten ohne direkten Auftrag, werden die Einlagerungs- und die Entnahmeprozesse aus den verschiedenen Lagerplätzen beschleunigt und flexibilisiert.

Das Projekt ist Teil des EffizienzClusters LogistikRuhr, das vom Bundesministerium für Bildung und Forschung gefördert wird. Projektpartner sind neben dem Lehrstuhl für Transportsysteme und Logistik auch der Lehrstuhl für Mechatronik, sowie zahlreiche Industriepartner. Mit der Entwicklung eines Regalbediengeräts auf Basis der SGP sind Leistungszuwächse sowie Kosteneinsparungen realistisch erreichbar. Die preiswerteren, neuartigen Regalbediengeräte werden somit zunehmend auch bei den vornehmlich kleinen und mittelständischen Unternehmen der Intralogistik eingesetzt werden können. Diese werden auch die beliebige Konfigurierbarkeit des Lagerraums und die räumliche Flexibilität zu schätzen wissen. Zudem werden die notwendigen Softwareentwicklungen zur Steuerung der Hardware ebenfalls von leistungsstarken und innovativen Unternehmen übernommen werden. 
Die aktuellen Forschungsarbeiten auf dem Gebiet der Regalbediengeräte konzentrieren sich im Wesentlichen auf die Verbesserung der bestehenden Komponenten. Untersucht wird der Einsatz neuer Werkstoffe für Teilbereiche der Regalbediengeräte und die Nutzung von energieeffizienten Betriebsverfahren. Die dabei zu erreichenden Leistungen sind an die Grenzen der Technik gestoßen.

Das SGP-Regalbediengerät beruht dagegen auf einer neuartigen Technik, die noch nie im Logistikbereich eingesetzt wurde. Dabei wird das klassische Regalbediengerät durch eine Plattform, die an acht vorgespannten Seilen eingespannt ist, ersetzt. Die Plattform wird mithilfe von acht Motoren, die unter Einsatz modernster Regelungsverfahren computergesteuert werden, bewegt. Es werden Kunstfaserseile verwendet, die sieben Mal leichter als die konventionellen Stahlseile sind. Dieser einfache Aufbau führt dazu, dass nur wenig Masse bewegt werden muss. Die Folge ist, dass die bereitgestellte Antriebsleistung weitgehend für die Bewegung der Nutzlast genutzt wird. Mithilfe des SGP-Regalbediengeräts werden höhere Geschwindigkeiten, Beschleunigungen und infolgedessen höhere Umschlagsleistungen erreicht. Außerdem spielt die Energieeffizienz und die zu bewegenden Massen eine große Rolle bei ihrer Entwicklung.

\section{SYSTEMKOMPONENTEN UND SYSTEMAUFBAU}

In diesem Kapitel wird erläutert, auf welcher Basis die Systemkomponenten festgelegt wurden. Diese hängen sehr stark von dem Aufbau des Systems ab.

Die Grundlagen zum praktischen Einsatz von seilbasierten SGPs stammen aus den DFG-Projekten SEGESTA (Seilgetriebene Stewart-Plattformen in Theorie und Anwendung) und ARTIST (Arbeitsraumsynthese seilgetriebener Parallelkinematikstrukturen) [Mec12]. Diese Projekte werden an der Universität Duisburg-Essen am Lehrstuhl für Mechatronik durchgeführt.

Nach der Durchführung von Simulationen und Berechnungen der Kräfte wurde festgestellt, dass die wirkenden Kräfte am Rand des Regales bei konventioneller Seilanordnung sehr hoch sind. Mithilfe einer neuen Seilanordnung wurde der Arbeitsraum vergrößert und die Zugkräfte verringert. Das führt zur weniger benötigter Motorleistung und besseren Arbeitsräumen. Außerdem weist die neue Seilanordnung mehr Systemstabilität auf.

In Abbildung 1 lässt sich die neue Anordnung der Seile erkennen. Hierbei werden die Seile gekreuzt angeordnet; wobei die Seile, die von den unteren Motoren angetrieben werden, oben an der Plattform befestigt sind. Die Seile sowohl in der YZ-Ebene als auch in XY-Ebene sind gekreuzt angeordnet. Mithilfe dieser Anordnung werden der Arbeitsraum und die Steifigkeit der Plattform vergrößert. Dies führt zu einer effektiveren Ausnutzung des Arbeitsraums.
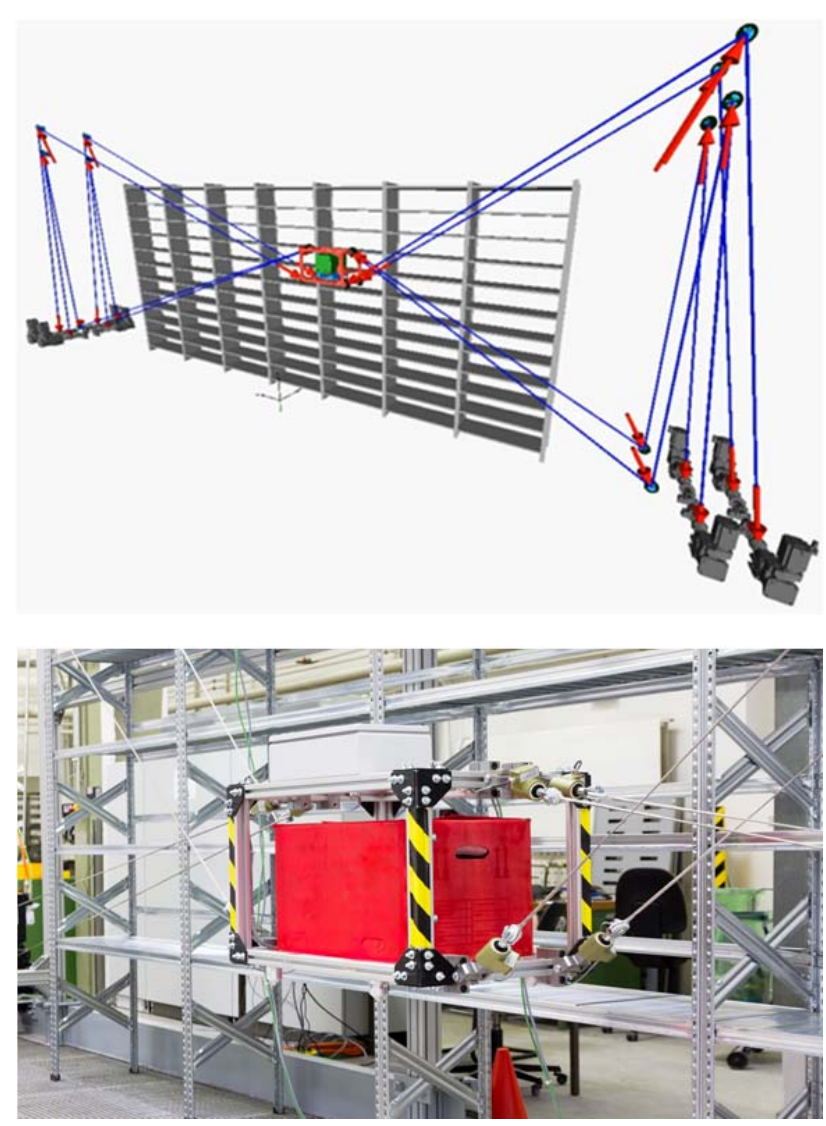

Abbildung 1. Oben: Übersicht des SGP-RBGs mit gekreuzter Seilanordnung[Mec12]. Unten: Die StewartGough-Plattform vor dem Regal, RESOURCE UND COPYRIGHT (C) Vom Institut für Produkt Engineering (IPE) der Universität DUISBURGESSEN

\section{ENTWICKLUNG EINES KONZEPTS ZUM LAGER- MANAGEMENT MIT OPTIMIERUNGSANSÄTZEN}

Die logische Steuerung des Regalbediengerätes wird von einer Software vorgenommen, die strikt modulbasiert programmiert wurde. Zentrales Softwaremodul der Steuerung ist das „RBG-Modul“. Hier werden die auszuführenden Lagerungsaufträge in eine Reihenfolge gebracht und in Routen für die Ansteuerung der Stellmotoren umgesetzt.

Das „RBG-Modul“ (siehe Abbildung 2) steuert das SGP-Regalbediengerät. Es stellt dafür die Ein- und Auslagerungsaufträge zu Einzel- oder Doppelspielen zusammen. Das Modul unterscheidet dabei "verspätete Auslagerungsaufträge", normale Auslagerungsaufträge, "Einlagerungsaufträge mit Priorität", "Auslagerungsaufträge mit Priorität" und normale Einlagerungsaufträge. Es reagiert auf die jeweilige Kombination unterschiedlich.

Ziel des Moduls ist eine möglichst schnelle Abarbeitung aller Aufträge durch die Minimierung von Fahrwegen. Es bietet die Möglichkeit durch Umlagerung von Be- 
hältern zu optimieren oder die Plattform an eine vorteilhafte Warteposition zu steuern. Das RBG-Modul bedient sich zur Ausführung seiner Funktionen zwölf anderer Module sowie diverser Untermodule, die es zustandsabhängig aufruft. rechnung der anzusteuernden Plätze greift das TSP-Modul auf eine sogenannte „Entfernungsliste“ zurück. Darin gespeichert sind die Entfernungen aller Fächer zueinander.

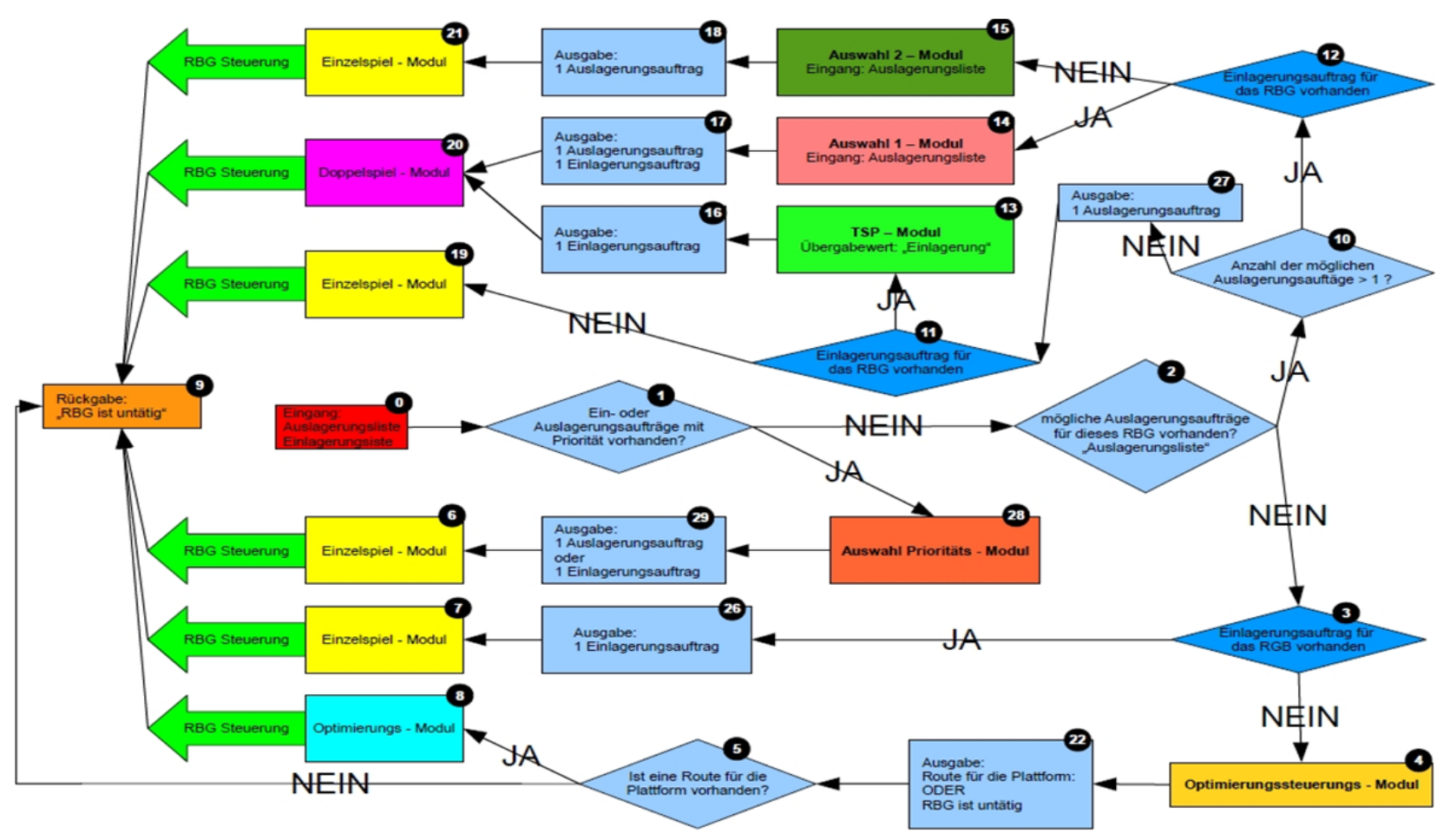

Abbildung 2. RBG-Modul

Eines der wichtigsten Module ist dabei das „TSPModul“ (siehe Abbildung 3). Das „TSP-Modul“ wählt aus allen zur Verfügung stehenden Fächern zu bestimmten Aufträgen genau nur jeweils die aus, bei deren Ausführung ein relativ kurzer Fahrweg entsteht. TSP steht für „Traveling Salesman Problem“ [Gut04]. Die Funktionsweise ist heuristisch und beruht auf der Annahme, dass eine gesamte Wegstrecke, die mehrere Stationen hat, dann besonders kurz ist, wenn möglichst viele Stationen beteiligt sind, die jeweils möglichst nah beieinander liegen. Das Modul arbeitet auf „Fachbasis“. Das bedeutet, dass es nicht direkt einen Auftrag auswählt, sondern es sucht nur aus einer Liste mit Fachnummern ein bis vier Fachnummern aus, die zu ein bis vier Aufträgen gehören. Ist eine Fachnummer bestimmt, ergibt sich daraufhin der damit verbundene Auftrag. Das Modul kann mit einem von drei Übergabewerten gestartet werden und liefert entsprechende Rückgabewerte. Vorteil der hiesigen Vorgehensweise ist der sehr begrenzte Rechenaufwand von maximal 4 Rechnungen, die von der Lagergröße und der Anzahl der möglichen Aufträge unabhängig sind.

Für jede Route sind drei Datenbankabfragen zur Längenberechnung nötig, daher ist es sinnvoll die Anzahl der zu berechnenden Routen konstant gering zu halten, unabhängig von der Anzahl der möglichen Aufträge. Zur Be-
Alternativ kann die Entfernungsliste auch die Zeiten speichern, die benötigt werden um die Plattform von einer Position zu einer anderen zu fahren.

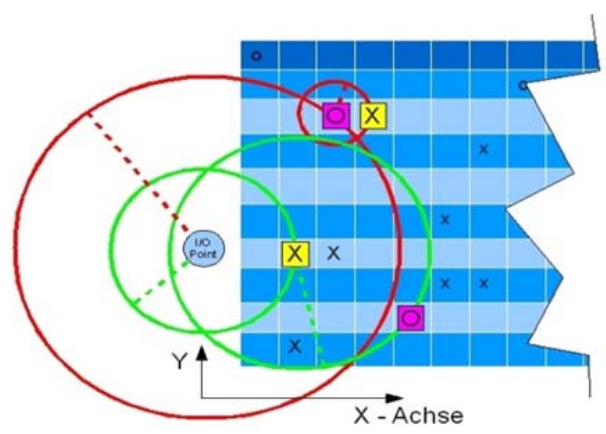

Abbildung 3. TSP-Modul

Es ist auch möglich z. B. Plätze, die aufgrund der technischen Ausgestaltung des SGP-Regalbediengeräts besonders gut zu erreichen sind, in der Entfernungsliste durch einen Abschlag attraktiver zu machen. Damit würden diese Fächer öfter angesteuert werden. Die Entfernungsliste wirkt wie ein Auswahlfilter für die Bewertung von Routen und erlaubt es so auf die neue Hardware des RBG optimal angepasste Routenkonzepte umzusetzen. 
Zeiten, in denen keine Ein- oder Auslagerungen durchgeführt werden müssen, eignen sich gut, um die Positionen von Lagergütern im Regal zu verändern, um es für kommende Aufträge zu optimieren. Diese Aufgabe wird vom „Optimierungssteuerungs-Modul“ (siehe Abbildung 4) ausgeführt. Ziel ist es dabei, in Abhängigkeit von der zur Verfügung stehenden Zeit, sowie des Lagerfüllstandes eine Verkürzung der Spielzeiten künftiger Aufträge zu realisieren. Die Optimierung erzeugt als Resultat eine Güterstruktur im Regal, die der einer ABCZonenlagerung ähnelt. Wenn zukünftige Aufträge bekannt sind, werden die nicht benötigten Güter vom E-/A-Punkt entfernt, um Platz für bald angeforderte Auslagerungen zu schaffen. Das entspricht im Geiste der Anforderung an eine Zonenlagerung, wo selten gebrauchte Güter in der CKlasse weit weg vom E-/A-Punkt eingelagert werden. Die Vorgehensweise erlaubt die Vorteile einer Zonenlagerung ohne eine dafür sonst nötige aufwendige Berechnung zur Klassifizierung, mit den Vorteilen der chaotischen Lagerung, mit der das RBG-Modul arbeitet, zu kombinieren. legt und im Gegenzug ein Gut, dessen Auslagerung nicht absehbar ist, aus der Nähe des E-/A-Punkts entfernt. Aufgabe zwei ist es innerhalb eines Radius $\mathrm{R}$ um den E-/APunkt immer einige freie Plätze für kommende Einlagerungen zur Verfügung zu stellen. Das Modul orientiert sich bei der Aufgabenauswahl am Lagerfüllstand innerhalb des Radius R um den E-/A-Punkt. Ziel des Moduls ist es, in Abhängigkeit vom Zustand des Regals, kommende Aus- und Einlagerungen gleichermaßen zu begünstigen.

In Abhängigkeit des Lagerfüllstandes können vom „Optimierungssteuerungs-Modul“ auch spezialisiertere Module gestartet werden, wie das „Freiplatz OptimierungModul“.

Das Modul verlagert Güter, die bis zu einem definierten Zeitpunkt nicht ausgelagert werden sollen, möglichst weit weg vom E-/A-Punkt. Dafür entsteht nahe am E-/APunkt ein freier Platz.

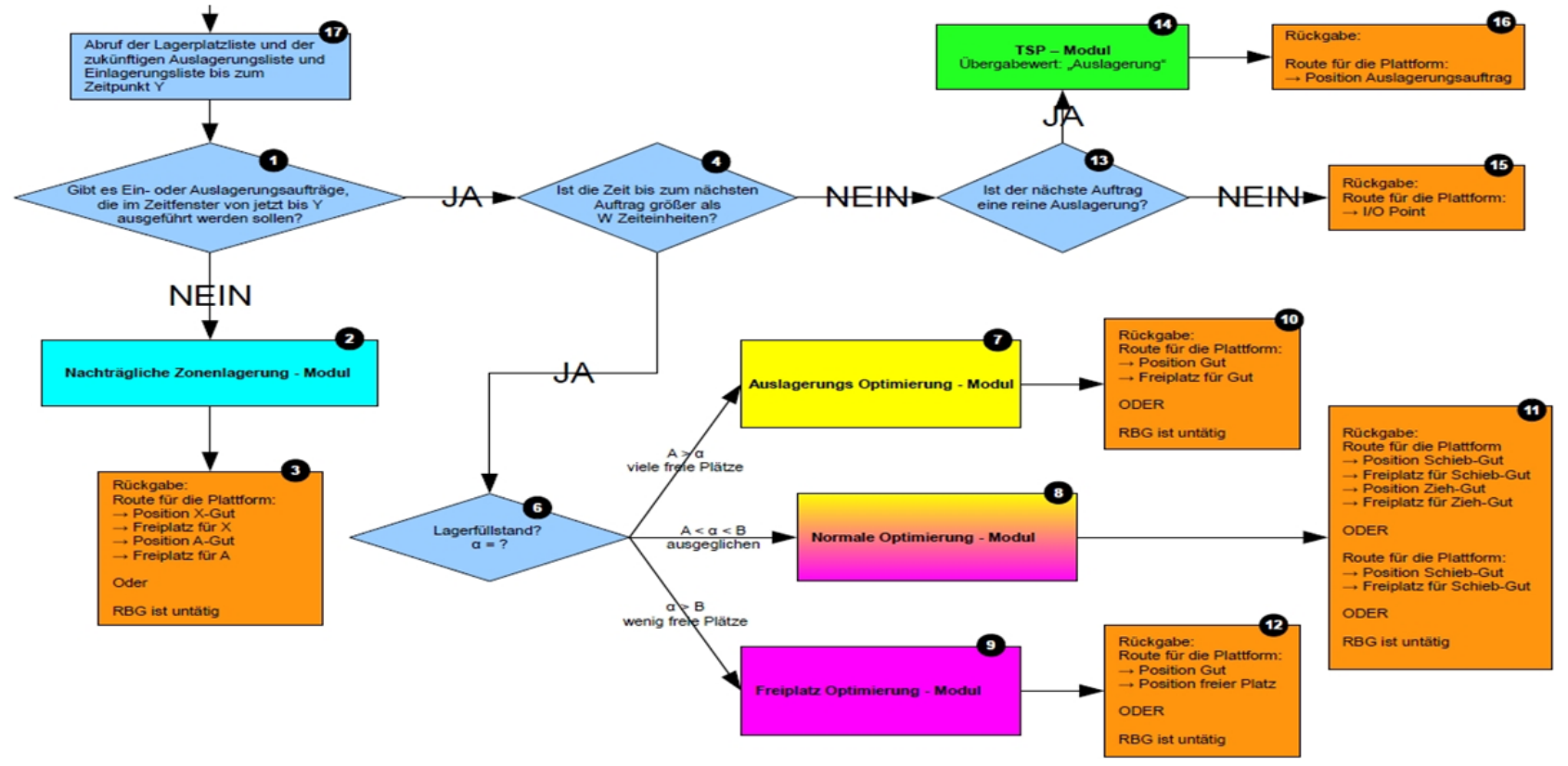

Abbildung 4. Optimierungssteuerungs-Modul

Das „Optimierungssteuerungs-Modul“ ist ähnlich wie das „RBG-Modul“ ein übergeordnetes Steuerungsmodul, das sich zur Erfüllung seiner Aufgaben diverser weiterer Module bedient. Dieses Modul wird dann aktiviert, wenn gerade kein Ein- oder Auslagerungsauftrag vorhanden ist, der ausgeführt werden kann. Es bewertet die Auftragslage sowie den Füllstand des Regals. Das Modul wählt je nach Bedarf die auszuführenden, spezialisierten Module zur Optimierung aus.

$\mathrm{Zu}$ den spezialisierten Optimierungsmodulen gehört das „Normale Optimierung-Modul“. Das Modul hat zwei Aufgaben. Aufgabe eins besteht darin zwei Güter im Regal zu vertauschen. Dabei wird ein Gut, das demnächst ausgelagert werden soll, in Richtung des E-/A-Punkts ver-
Das „Auslagerungs-Optimierung-Modul“ wählt aus einer Liste der in den nächsten $\mathrm{T}$ Zeiteinheiten bevorstehenden Auslagerungen ("Baldige Auslagerungsliste") den Auftrag aus, der sich am weitesten vom E-/A-Punkt entfernt befindet. Dieses Gut wird nach vorne in Richtung E-/A-Punkt gezogen. Das Modul berücksichtigt, ob es mehrere identische Güter im Regal gibt, auf die derselbe Auslagerungsauftrag passt. Es optimiert nur dann für einen solchen Auftrag, wenn sich das auszulagernde Gut nicht ohnehin schon innerhalb eines Radius $\mathrm{R}$ um den E-/A-Punkt befindet.

Für längere Zeiten ohne Aus- oder Einlagerungsaufträge wurde zusätzlich das „Nachträgliche Zonenlagerung-Modul“ entwickelt (siehe Abbildung 5). Es setzt vo- 
raus, dass alle Güter im Lager nach den Verfahren der ABC-Analyse in Klassen eingeteilt werden können.

Das Modul sortiert die Güter im Regal um. Dabei sollen B- und C-Güter aus der Zone, die für A-Güter bestimmt ist, entfernt werden. A-Güter, die sich in einer Zone außerhalb der A-Zone befinden, werden in die A-Zone verbracht. Das Modul überprüft dabei, ob freie Plätze in der jeweiligen Ziel-Zone verfügbar sind. Je nach Zustand des Regals werden entweder Doppelspiele oder Einzelspiele gefahren.

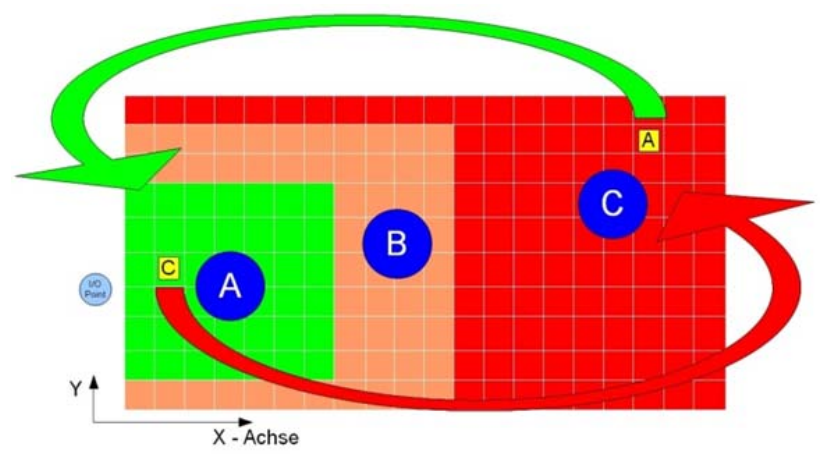

Abbildung 5. Optimierungssteuerungs-Modul

\section{FAZIT}

Die Anzahl der gelieferten Regalbediengeräte im Jahr 2007, 2008 und 2009 in Deutschland und international nach VDMA hat gezeigt, dass die Nachfrage nach Regalbediengeräten mit einer Gerätehöhe ab $6 \mathrm{~m}$ und einer Nutzlast unter $100 \mathrm{~kg}$ hoch ist [VDMA09]. Im Rahmen einer Marktanalyse wurden sechs konventionelle Regalbediengeräte unter die Lupe genommen und mit dem SGP-RBG verglichen. Dabei wurde festgestellt, dass alle konventionellen Regalbediengeräte auf eine bestimmte Höhe beschränkt sind. Die typische Höhe, die von Regalbediengeräten erreicht wird, ist $12 \mathrm{~m}$. In Ausnahmefällen kann ein Regalbediengerät eine Höhe von 24 m erreichen. Im Unterschied dazu ist das SGP-RBG in der Höhe unbeschränkt. Ein weiterer Vorteil des SGP-RBGs ist die hohe Dynamik (hohe Geschwindigkeit und Beschleunigung), die von anderen Regalbediengeräten nicht erreicht werden kann. Konventionelle Regalbediengeräte werden so leicht wie möglich gebaut, um die bewegte Masse zu reduzieren. Aufgrund der schlanken Konstruktion können hohe Beschleunigungs- und Geschwindigkeitswerte bereits einen Aufbau von Schwingungen des Tragwerks begünstigen, welche auf das damit verbundene Fahrwerk übertragen werden. Ebenso wirkt sich vor allem beim Brems- und Beschleunigungsvorgang die Massenträgheit des Mastes nachteilig auf die Stabilität des Regalbediengeräts aus, was den Einsatz eines Regelungshilfsantriebs unerlässlich macht. Beim SGP-RBG werden diese Probleme vermieden, da der Mast und der Hubwagen durch eine Plattform ersetzt werden.
Das hier vorgestellte an die Anforderungen der neuen SGP-RBG angepasste Softwarekonzept zum Lagermanagement umfasst ein großes Spektrum an Funktionen. Es stellt sowohl Mechanismen zur Auswahl, Reihenfolgebestimmung und Platzvergabe für eintreffende Ein- und Auslagerungsaufträge zur Verfügung, als auch Methoden zur Optimierung der Lagerplatzbelegung, wenn das RBG untätig ist. Die verwendeten Methoden zur Bestimmung der Route für die Plattform sowie für die Umlagerung in Zeiten ohne Aufträge sind durch ihren geringen Rechenaufwand gekennzeichnet. Damit leistet der Algorithmus durch Schonung von Datenverarbeitungsressourcen ebenfalls einen Beitrag zum Umweltschutz und verringert die nötigen Investitionen in die EDV-Infrastruktur.

\section{LITERATUR}

[Sal11] Salah, Bashir; Ramadan, Muawia; Noche, Bernd: Travel Time Analysis of Stewart-Gough Platform in Automated Storage and Retrieval System. IEEE International Conference on Automation and Logistics, ICAL 2011, Chongqing, China, 15-16 August, 2011; 01/2011

[Mec12] Universität Duisburg-Essen, Lehrstuhl für Mechatronik: Seilgetriebene Stewart -Plattformen in Theorie und Anwendung. 31.03.2007, http://www.unidue.de/segesta/ Abruf am 15.Mai, 2012.

[Gut04] Gutin, Gregory; Punnen, Abraham P.: The Traveling Salesman Problem and Its Variations. Volume 12, SpringerVerlag Gmbh , - ISBN: 0-306-48213-4

[VDMA09] VDMA-Statistikdatenbank (VDMA:Verband Deutscher Maschinen-und Anlagenbau e.V.). 